\title{
CHEMICAL EVOLUTION OF CNO ABUNDANCES
}

\author{
M. GAVILÁN AND M. MOLLÁ \\ Departamento de Fúsica Teórica \\ Universidad Autónoma de Madrid, Cantoblanco, E-28049 Madrid, Spain
}

\begin{abstract}
New low and intermediate mass star yields recalculated by Buell (1987) are evaluated by using them in a Galactic Chemical Evolution model. We analyze their effects on CNO elemental abundances
\end{abstract}

\section{Introduction}

Stellar yields for low and intermediate mass (LIM) stars have been recalculated by Buell (1997), who, taking into account the effects of the convective dredge up and the Hot Bottom Burning (HBB), incorporates the most recent improvements in the TP-AGB processes.

The aim of this job is to evaluate these new yields for LIM stars by using them as input in a Galactic Chemical Evolution (GCE) model. In particular, we analyze if the contribution of these stars is enough to justify the observed behavior of nitrogen vs. oxygen.

\section{Stellar structure for LIM stars during the TP-AGB phase}

The evolution of a LIM star during the TP-AGB phase is dominated mainly by two processes: The third dredge up events and the Hot Bottom Burning (HBB). The first ones affect stars with masses between 2 $\mathrm{M}_{\odot}$ and $4 \mathrm{M}_{\odot}$, by increasing the abundance of carbon. In stars more massive than $4 \mathrm{M}_{\odot}$, the HBB produces an abrupt increase of nitrogen and a similar decrease of carbon. In Figure 1 we show the ejected masses of ${ }^{12} \mathrm{C}$ and ${ }^{14} \mathrm{~N}$ as a function of the stellar mass $M_{*}$, for $Z=\mathrm{Z}_{\odot}$, compared with those from RV, where we see that the described behaviour for both elements does not appear since those authors had not taken into account the transformation from carbon in nitrogen during the HBB phase. In fact, nitrogen shows a variation around $4 \mathrm{M}_{\odot}$ for RV but it does not increase for larger stellar masses. 


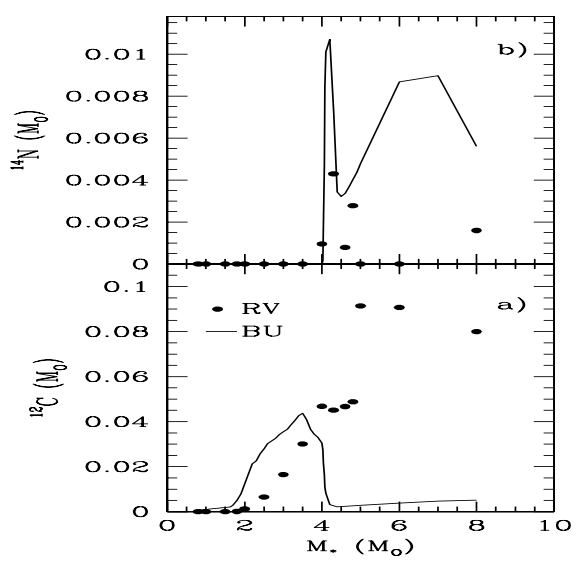

Figure 1. Carbon and nitrogen ejected for LIM stars for $Z=0.02$. Solid lines are BU yields, points are from RV.

\section{Computed models}

In order to compare the results obtained with these new stellar yields with those produced by other sets, we have also used those from RV for LIM stars. For massive stars, we have chosen those from Woosley \& Weaver (1995; hereafter WW), who joined explosive nucleosynthesis to the themselves pre-supernova yields for metallicities between $\mathrm{Z}=0$ and $\mathrm{Z}=\mathrm{Z}_{\odot}$; and those from Portinari, Chiosi \& Bressan (1998; hereafter PCB), who obtained the yields for the Padova group metallicities for $\mathrm{M}>8 \mathrm{M}_{\odot}$ and considered the mass loss by stellar winds, the influence of the metallicity during the stellar evolution and the explosive nucleosynthesis. By combining these sets, we run four models: BU-PCB, BU-WW, RV-PCB, RV-WW.

On the other hand, since the origin of nitrogen in massive stars is not clear, we have also run the same four models but considering $\mathrm{N}$ as primary, doing a total of eight models. They are used in the GCE model, as implemented with the multiphase chemical evolution model (Ferrini et al. 1992; Mollá, Díaz \& Ferrini 2002).

\section{Results}

Once the Galaxy is modeled, we study the behaviour of the CNO elements. Results referring to gas structure, time evolution and radial gradients are analyzed in Gavilán, Buell \& Mollá (2003)

We study the relation $\log (\mathrm{N} / \mathrm{O})$ vs. $12+\log (\mathrm{O} / \mathrm{H})$ in Figure 2 for models with nitrogen as secondary (panel a) or primary (panel b). No 

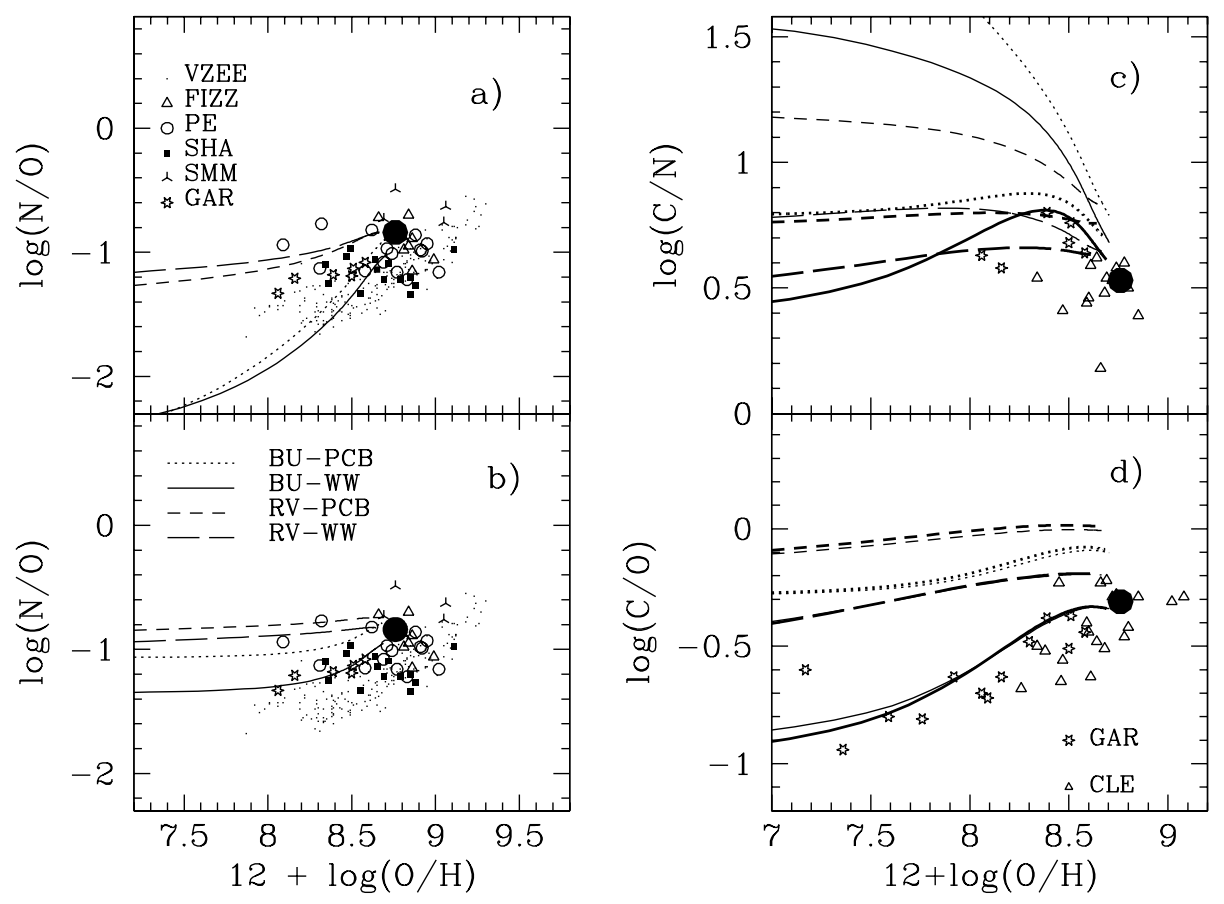

Figure 2. Left: The relation $\log (\mathrm{N} / \mathrm{O})$, vs. $12+\log (\mathrm{O} / \mathrm{H})$ whith a) Secondary nitrogen and b) Primary nitrogen. Data are from Fitzsimmons et al. (1992, FIZZ), Garnett et al. (1999, GAR), Peimbert (1979, PE), Shaver et al. (1983, SHA), Smartt et al. (2001, SM), and Van Zee (1998, VZEE). Right: C) The relation $\log (\mathrm{C} / \mathrm{N})$ and d) $\log (\mathrm{C} / \mathrm{O})$ vs. as $12+\log (\mathrm{O} / \mathrm{H})$. Data are from Garnett et al. (1995; 1999, GAR), and from Clegg et al. (1981, CLE). Thick lines mean N primary while thin lines are models with $\mathrm{N}$ as secondary. The large filled dot is the observed solar value.

model with $\mathrm{N}$ as secondary reproduces the observed trend. Models fit the data better if nitrogen is considered primary, mostly the BU-WW model. In this case, the model line has a slope close to zero at the beginning, and then develops a positive slope, caused by the behaviour of nitrogen yields for stars of different stellar masses: when stars with masses larger than $4 \mathrm{M}_{\odot}$ begin to evolve, the nitrogen production suffers a great change. In the RV models this change cannot be seen because yields of $\mathrm{N}$ are quite uniform.

The relation $\mathrm{C} / \mathrm{N}$ vs. $\mathrm{O} / \mathrm{H}$ is shown in Figure 2 panel c), where the 8 models are included. Again, data are better reproduced when nitrogen is primary -thick lines- than when nitrogen is secondary -thin lines. In particular, Model BU-WW is able to reproduce the variable 
slope shown by the data, due to the evolution of LIM stars with masses close to $4 \mathrm{M}_{\odot}$, after the primary nitrogen ejected by massive stars. The same effect can not be obtained either with secondary nitrogen or with primary nitrogen in other yields. The effect of HBB and third dredge up processes is seen in this figure as the decreasing of $\mathrm{C} / \mathrm{N}$ at high oxygen abundance. This means that nitrogen appears while $\mathrm{C}$ is not ejected yet by the less massive stars,

Finally, in Figure 2, panel d), we show the relation of carbon abundance with the oxygen abundances, which does not change with the different origin of the nitrogen. Again Model BU-WW fits the HII region observations better than the others. Model BU-PCB shows the same trend but with a higher absolute value. This variable slope of $\mathrm{C} / \mathrm{O}$ vs. $\mathrm{O} / \mathrm{H}$ is explained here by the normal evolution of LIM stars, instead of using metallicity depending yields.

\section{Conclusions}

We have used new stellar yields based on a detailed modeling of the TP-AGB phase, which affects the nitrogen and carbon production directly, in a model of Chemical Evolution of the Galaxy disk. The evolution of the solar neighborhood obtained with this model including these yields for LIM stars and WW yields for massive stars, is able to reproduce the HII regions and stellar data. The trend of $\mathrm{C} / \mathrm{N}$ vs. $\mathrm{O} / \mathrm{H}$ is reproduced without using metallicity depending yields.

\section{References}

Buell J.F., PhD Thesis, 1997, U. of Oklahoma (BU)

Clegg R.E.S., Lambert D.L., Tomkin J., 1981, ApJ, 250, 262

Ferrini F., Matteucci F., Pardi C., Penco U., 1992, ApJ 387, 138 (FMPP92)

Fitzsimmons A., Dufton P.L., Rolleston W.R.J., 1992, MNRAS, 259, 489

Garnett D.R., Shields G.A., Peimbert M., Torres-Peimbert S., Skillman E.D., Dufour R.J., Terlevich E., Terlevich R.J., 1999, ApJ, 513, 168

Garnett D.R., Skillman E.D., Dufour R.J., Peimbert M., Torres-Peimbert S., Terlevich R., Terlevich E., Shields G.A., 1995, ApJ, 443, 64

Maeder A., 1992, A\&A, 264, 105

Mollá M., Díaz A.I., Ferrini F., 2003, ApJ, submitted

Peimbert M., 1979, in I.A.U. Symp. 84, The Large Scale Characteristics of the Galaxy, Ed. W. B. Burton, (Reidel: Dordrecht), p.307

Portinari L., Chiosi C., Bressan A., 1998, A\&A, 334, 505 (PCB)

Renzini A., Voli. M., 1981, A\&A, 94, 175 (RV)

Shaver P.A., McGee R.X., Newton L.M., et al., 1983, MNRAS, 204, 53

Smartt S.J., Rolleston W.R.J., 1997, ApJ, 481, L47

van Zee L., Salzer J.J., Haynes M.P., 1998, ApJ, 497, L1

Woosley S.E., Weaver T.A., 1995, ApJS, 101, 181 (WW) 